\title{
Direct Observation of Heavy Metals and Impurities in Minerals Using Z-contrast Imaging
}

\author{
Huifang $\mathrm{Xu}^{1 *}$ \\ 1. Department of Geoscience, University of Wisconsin-Madison, Madison, WI, USA. \\ * Corresponding author: hfxu@geology.wisc.edu
}

Understanding interface structures, nano-precipitates, vacancies, impurities, and adsorbed atoms on mineral surfaces are important to elucidate formation mechanism and reactions of minerals in the earth environments. Aberration-corrected Z-contrast imaging can provide chemical images with sub- $\AA$ resolution. Z-contrast images are HAADF images with atomic resolution. Multiple diffraction effects that appear in high-resolution transmission electron microscopic (HRTEM) images can be eliminated or minimized in Z-contrast images, because Z-contrast imaging uses non-coherent elastically scattered electrons at high scattering angle. We can obtain positions of atoms directly over a large range of thickness, with Z-contrast to help distinguish columns of different atoms and their occupancies along the beam direction. Interface structures and crystal structures of nano-minerals and nano-precipitates can be solved by combining the Z-contrast imaging and ab-initio calculation using density functional theory (DFT) methods. Vacancies, impurities, adsorbed heavy atoms can be also revealed directly [1-2]. Zcontrast images of the Fe-oxyhydroxides show ordered FeOOH proto-goethite nano-domains intergrown with nanophase goethite. The FeOOH nanophase is a precursor to the goethite. DFT calculations indicate that goethite is more stable than proto-goethite. Our results suggest that ordering between Fe and vacancies in octahedral sites result in the transformation from feroxyhyte to goethite through a proto-goethite intermediate phase. Combining Z-contrast images and TEM-EDS reveals that arsenate $\left(\mathrm{AsO}_{4}{ }^{3-}\right)$ tetrahedra are preferentially adsorbed on the proto-goethite (001) surface (Fig.1). Vacancy ordering in Fe-bearing forsterite olivine was observed. The phenomenon may explain observed highly anisotropic diffusion in some Fe-bearing olivine minerals and more ordered structure analyzed at high-temperature in air. In a Sibearing magnetite, $\mathrm{Si}$ replaces $\mathrm{Fe}^{3+}$ in tetrahedral sites of the magnetite structure and vacancies are introduced in the octahedral $\mathrm{Fe}^{2+}$ sites (Fig. 2). Nano-precipitates of Si-magnetite with composition of $\left(\square 0.5 \mathrm{Fe}^{2+}{ }_{0.5}\right)^{\mathrm{VI}}\left(\mathrm{Fe}^{3+}\right)^{\mathrm{VI}} \mathrm{Si}^{\mathrm{IV}} \mathrm{O}_{4}$ or $\gamma-\mathrm{Fe}_{1.5} \mathrm{SiO}_{4}$ occur in the magnetite [4].

\section{References:}

[1] Lee, S., Shen, Z., and Xu, H. American Mineralogist, 101 (2016), p. 1986.

[2] Xu, H., Shen, Z., and Konishi, H. Mineralogical Magazine 79 (2015), p. 377.

[3] Xu, H., Shen, Z., and Konishi, H. American Mineralogist, 99 (2014), p. 2196.

[4] Author thanks supports from NASA Astrobiology Institute, National Science Foundation, and US

Department of Energy. 

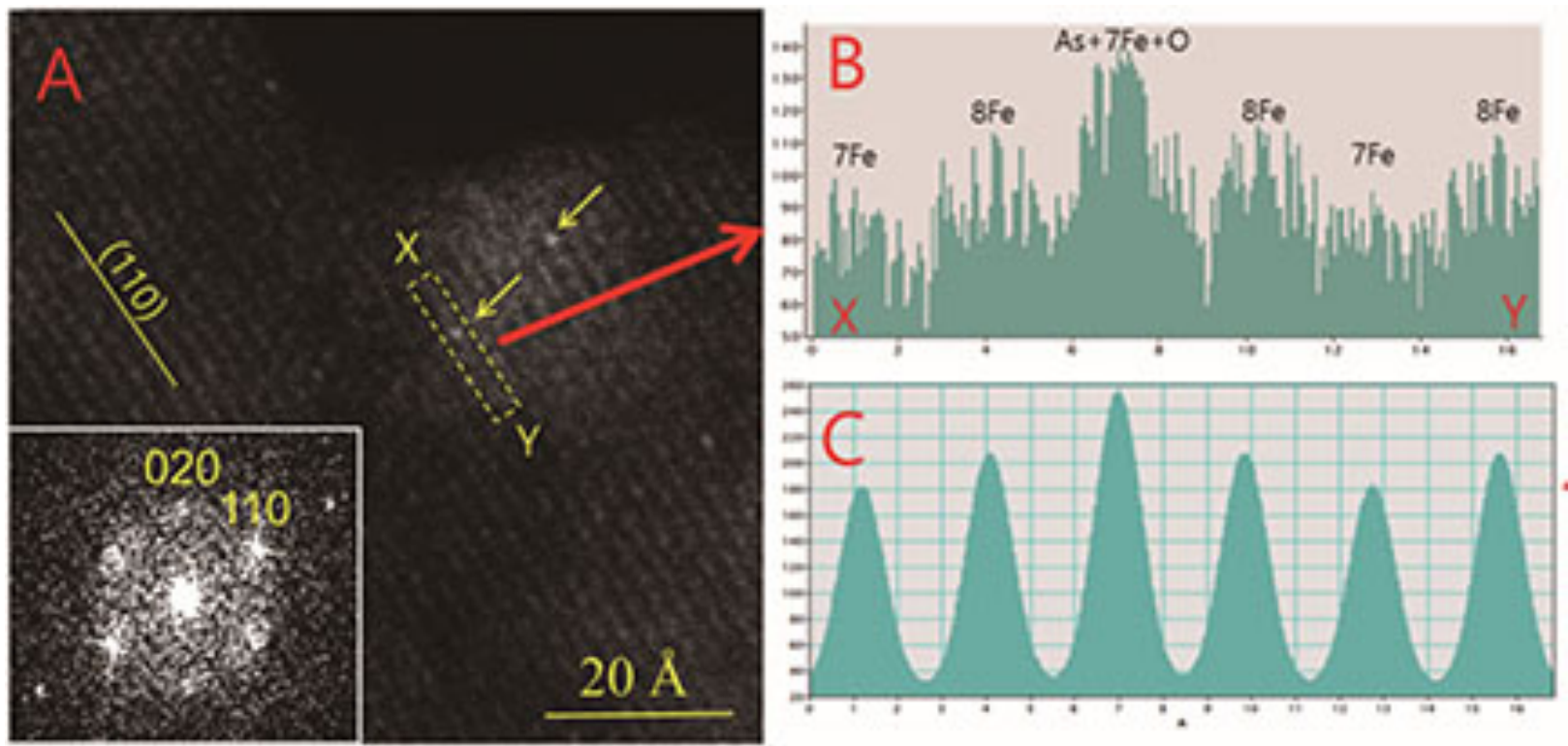

Figure 1. (A) a [001]-zone-axis Z-contrast image of proto-goethite, bright spots are positions of Fe atom columns. Very bright spots are adsorbed arsenic (As) atoms on the surface right above Fe; (B) the intensity profile of an outlined area from X to $\mathrm{Y}$; (C) the intensity profile of simulated Z-contrast image showing the peak with As atom above an Fe atom column (Lee at el., 2016).
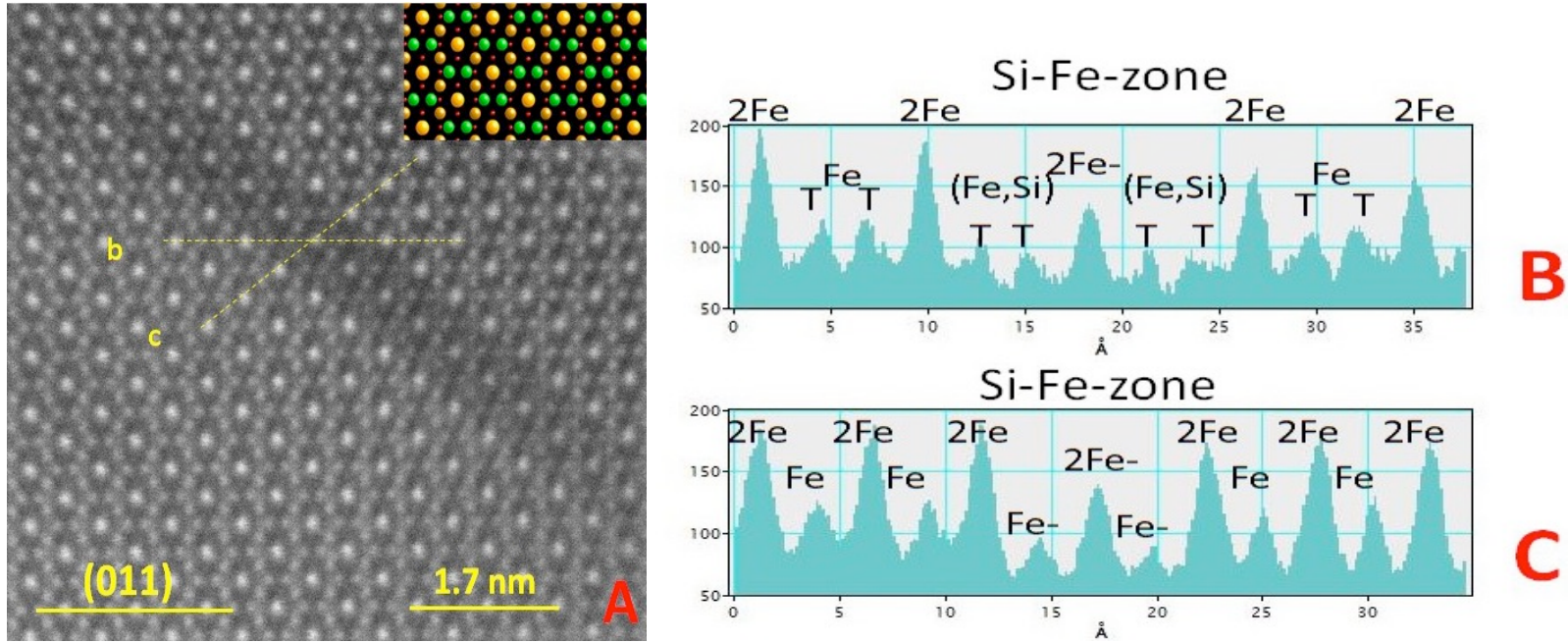

Figure 2. Z-contrast image showing a thin nano-precipitate with thickness of $2 \mathrm{~d}_{111}$. The precipitates are of the Si-magnetite phase, although both the host and precipitates have the same structure. Intensity line profiles can indicate occupancies of $\mathrm{Si}$ in tetrahedral sites $(\mathrm{T})$ and vacancies in octahedral sites (labeled by $2 \mathrm{Fe}$-, and Fe- (Xu et al., 2014). 